\title{
Designing an Object Tracker Self-Balancing Robot
}

\author{
${ }^{1}$ Yunus Çelik(0000-0002-3384-4213), *² Mahit Güneş(0000-0002-1552-3889)
${ }^{1}$ Karamanoğlu Mehmetbey University, Faculty of Engineering, Department of Electrical and Electronics Karaman, Turkey ycelik@kmu.edu.tr \\ ${ }^{2}$ Kahramanmaraş Sütçü İmam University, Faculty of Engineering, Department of Electrical and Electronics Kahramanmaraş, \\ Turkey mgunes@ksu.edu.tr
}

Geliş Tarihi: 04.12.2017～Kabul Tarihi: 31.05.2018

\begin{abstract}
Real-time robots are quite common in our daily life. These robots are working as a part of the process in industry or a medical assistance in hospitals to serve humanity. Designing the robots according to the desired referent and making the given tasks with high accuracy makes them more and more popular in these days. In this work, the designed two-wheeled balancing robots with integrated camera track object autonomously. This work has two important stages. The first stage is about balancing the robot with the angle information taken from IMU sensor and implementation of PID control. IMU sensors create lots of noisy signals because of its natural structures. Kalman filter was used to denoise these noisy signals to have a smooth signal for a better balance control. The second stage is about image processing and objects recognition. This section was completed by using Matlab Image Processing Toolbox which can be used Arduino microcontroller board synchronously. In this section, algorithm infers motion information of objects. Motors were controlled according to motion information of moving objects. In the end, an object tracker self-balance robot was constructed. Balance control of the robot was managed by PID controller and accelerometer signals were denoised by a Kalman Filter. It was clarified that using PID controller and Kalman Filter together have a positive effect to balance the robot on the desired angle.
\end{abstract}

Keywords: Balance robot, Kalman filter, Object recognition

\section{INTRODUCTION}

Thanks to the latest technological developments, image processing and control methods can be applied successfully in a very short time. Advanced computers play an important role in shortening the process and getting successful results. Camera-assisted robots are starting to take their place in daily life. These robots are helping us in almost every step of our daily works so image processing techniques and control methods are an indispensable part of fulfilling given tasks for the robots. Most of unmanned aerial vehicles and vehicles that draw a specific route without the need for a driver were designed by using image processing techniques and control techniques. Some of these vehicles have the ability to destroy the target in case of needed and they are commonly used in military defence systems. It is possible to say that countries having such technologies are more powerful in terms of military and economy. Moreover, they are in a better position than other countries in terms of prestige. That's why, countries invest more money for the defence industry day by day. Another usage area of real time robotic application is related to human transportation vehicles. Segway can be an example of a two-wheeled human transportation vehicle. It was invented by Kamen Segway in 2001. The working principle of Segway robot is quite similar to two-wheeled hoverboards and two-wheeled scooters.

By means of the angle information obtained from the acceleration sensor, the robot tries to keep the mass in upright position. When the robot is tilted by a user, it autonomously tries to reach upright position [1-4].During this process, the wheels turn into the side where the robot was tilted to keep the body upright. In this way robot moves to the side of tilt. Since the balance robots have a non-linear structure, the study of modelling and evaluating the success of control methods are quite useful to understand control theory. The robot was successfully designed by combining Kalman filter and PID control method. Several difficulties were encountered during the designing phase of the robot. The first one was about adjusting sensitively the centre of gravity in the middle of mass by taking into account the hardware weights. The mass of the robot was printed by a 3-D printer and hardware were attached carefully. The second difficulty was derived from noisy sensor signals. The difficulty was solved by using Kalman filter. Another problem was about tuning PID parameters. It was difficult to tune PID 
parameter automatically when the exact mathematical model of the system was unknown. This tuning has been done by trial and error method. The last difficulty was about detection of RGB objects in the environments having unstable amount of lights [5-7].

In this paper, it was aimed to point out the positive effect of usage of Kalman filter and PID control on balance robot. It was clarified that using these methods balance can be more stable and robust. The results and measurements of balance robot were presented in the result section [5-7].

\section{RELATED RESEARCHES}

In the literature, there are several balancing robot designs. These designs use different control methods and different mechanical models. Nonlinearity of these robots attract the researchers for designing and controlling. Karla and friends were heavily interested in the reducing of noise amount of IMU sensors [4, 8, 9]. In another work, Kao and friends designed a robot quite similar to this project. Raspberry Pi microcontroller process the PID phase and image processing phase together. David P. who is the inventor of N BOT combined accelerometer and gyroscope sensors to have more accurate signals. In another paper, Grasser and friends evaluated the performance of balance control by putting some weight on the robot [6-10]. In another work, Segway which is a human transporter vehicle have 5 IMU sensors inside. Three of the sensors are working actively and other two are backup sensors. This design can reach $20 \mathrm{~km} / \mathrm{h}$ speed. In another work, Linear Quadratic Regulator control was used to balance the robot named Gyro boy.[10-14].

While designing the robot, literature works helped a lot to get to know the working principle of balance robots. The necessary hardware was chosen carefully by looking at the previous studies.

\section{SYSTEM MODEL AND ARDWARE}

\subsection{System Model and Parameters}

Balance robot can be referred as two wheeled inverted pendulums in the literature. Operation logic of balance robot is so similar to the inverted pendulum. Figure 1 shows the physical model of two wheeled inverted pendulum and its parameters.

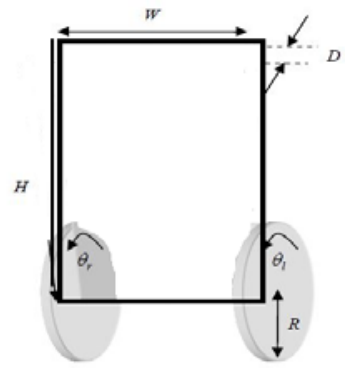

Figure 1. Physical Model of Balance Robot
$\mathrm{W}$ is the width of body, $\mathrm{D}$ is thickness of body, $\mathrm{H}$ is the length of body and $\mathrm{R}$ is the radius of the wheels. Figure 2 shows the top and side view of the body on coordinate the system.
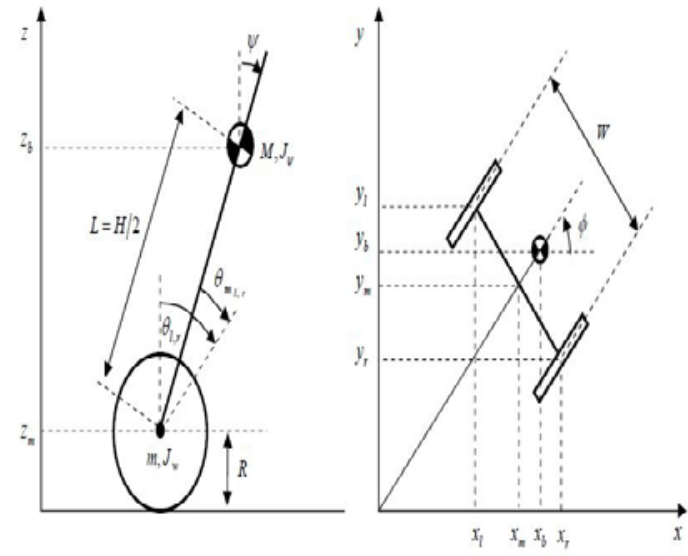

Figure 2. Top and Side View of Body

$\Psi$ is the balance angle of the body. $\theta_{\mathrm{l}, \mathrm{r} \text { is the wheels }}$ angle. $\phi$ is turning angles. Below table shows some parameters of robot.

Table 1. Physical Parameters of Robot

\begin{tabular}{|c|c|}
\hline $\begin{array}{c}\text { Parameter } \\
\text { s }\end{array}$ & Explanation \\
\hline $\begin{array}{c}\mathrm{g}=9,81 \mathrm{~m} / \mathrm{sn} \\
2\end{array}$ & $\begin{array}{c}\text { Gravitational } \\
\text { acceleration }\end{array}$ \\
\hline $\mathrm{m}=3$ grams & Weight of wheels \\
\hline $\mathrm{R}=4 \mathrm{~cm}$ & Radius of wheels \\
\hline $\mathrm{Jw}=\mathrm{mR} 2 / 2$ & Weight of robot \\
\hline $\mathrm{M}=1.5 \mathrm{~kg}$ & Width of body \\
\hline $\mathrm{W}=19 \mathrm{~cm}$ & Thickness of body \\
\hline $\mathrm{D}=9 \mathrm{~cm}$ & Length of body \\
\hline $\mathrm{H}=1 \mathrm{~cm}$ & Centre height of body \\
\hline $\mathrm{L}=\mathrm{H} / 2 \mathrm{~m}$ & \\
\hline
\end{tabular}

Motion equations of robot was derived from the above parameters [4, 9, 10, 12, 13].

$(\theta, f)=\left[1 / 2\left(\theta_{l}+\theta_{r}\right) R / W\left(\theta_{r_{-}} \theta_{l}\right)\right]$

$X m, Y m=\left(\not \& n d t, I_{q n d t}\right)=(R \& \cos \phi, R \& \sin \phi)$ 
$\left(X_{\text {left }}, Y_{\text {left }}, Z_{\text {left }}\right)=\left[\begin{array}{c}X m-\frac{W}{2} \sin \phi, Y m \\ +\frac{W}{2} \cos \phi, Z m\end{array}\right]$

$\left(X_{\text {right }}, Y_{\text {right }}, Z_{\text {right }}\right)=\left[\begin{array}{c}X m+\frac{W}{2} \sin \phi, Y m \\ -\frac{W}{2} \cos \phi, Z m\end{array}\right]$

$(X b, Y b, Z b)=\left[\begin{array}{c}X m+L \sin \psi \cos \phi, Y m+ \\ L \cos \psi \sin \phi, Z m+L \cos \psi\end{array}\right]$

\subsection{System Hardware}

The robot consists of a body, 2 motors, a motor driver, an IMU sensor and a camera. Component selection was carefully managed according to related work and simulation results.

\subsubsection{Motors}

Direct Current motors are both cheap and highperformance motors. Balance robot motors are $24 \mathrm{~V}, 500$ rpm DC motors with reductor. The reason why it was chosen a motor with reductor is that it helps to have a big momentum with low power.

Voltage range and RPM (Revolutions per Minute) range is enough to have a balance control of the robot $[9,10,12-$ 15]. Figure 3. Shows a picture of DC motor.

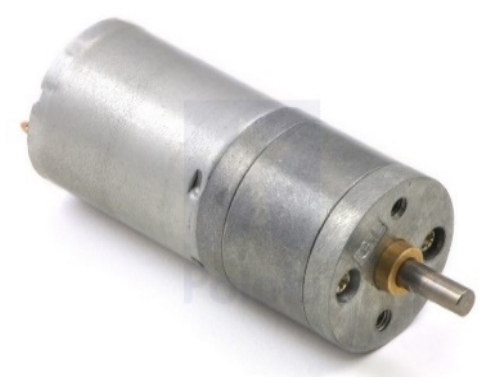

Figure 3. DC Motor Picture

\subsubsection{Motor Driver}

L298N is a motor driver which can drive two motors at the same time. It can perform in the temperature range from 20 to +135 . The input voltage of motor driver is $5-35 \mathrm{~V}$ and it can give max 2 A to drive a motor. It is important to connect the ground of motor driver and microcontroller ground at the same point because of $\mathrm{I}^{2} \mathrm{C}$ communication platform.
Motor speed is adjusted according to body angle, that's why the speed always prone to changes. Pulse with modulation (PWM) signals are used to drive motor in certain speed $[9,10,12,15]$. Figure 4 . Shows a picture of motor driver.

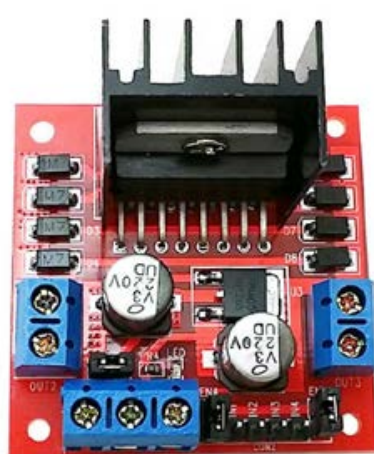

Figure 4. Motor Driver Picture

\subsubsection{Sensor}

Acceleration and gyroscope sensors give the main and most important parameters of robot. In this robot, IMU 6 axis sensor was used to get the mass angle. The disadvantage of the sensor is the amount of noise it creates. It is important to attach the sensor to a place away from the motors because of motor vibrations. The sensor can read accelerometer and gyroscope values for $\mathrm{x}, \mathrm{y}$ and $\mathrm{z}$ axis. It uses I2C protocol to communicate with microcontroller board up to $400 \mathrm{kHz}$.
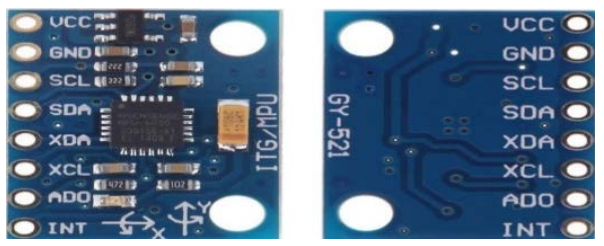

Figure 5. Gyro Sensor Picture

To overcome the noise ratio, Kalman filter was used. Sensor gives better and smoother results after filtering.

\subsubsection{Microcontroller}

Arduino microcontroller board uses ATmega328 processor attached over the card. Arduino is one of the most common microcontrollers for educational purposes. It is an open source platform which is easy to be programmed. In recent years, this microcontroller can operate with Matlab simultaneously. This feature is the key for the second part of our system. 6 digital pins of Arduino were used in this project. 4 pins were used to drive motor with HIGH, LOW to determine the rotation. Other 2 pins were PWM pins for analogue inputs. IMU sensor is also used 2 analogue pins $[9,10,12-15]$. 


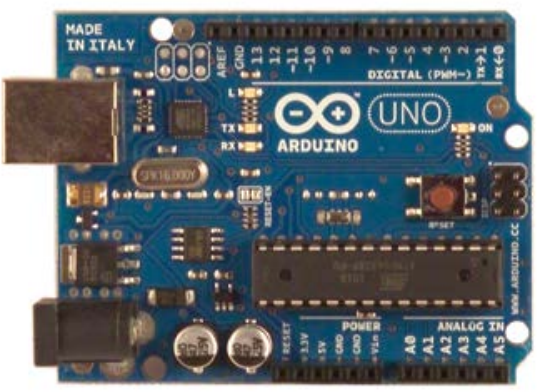

Figure 6. Microcontroller Picture

\subsubsection{Camera}

The camera used in the robot has 0.3 Megapixel and takes $640 \times 480$ pixel photos. The quality of the camera resolution is low because low-quality images are faster to process than high quality images.

\section{METHODS}

In this section, the methodology of control theory and image processing were presented.

\subsection{Kalman Filter}

Kalman Filter is an algorithm which recursively computes estimates of observed states over time [1]. It is also a mathematical equation which is used as a predictor[2]. As it is stated above, IMU sensor has lots of noise because of its sensitive structure. It can be affected by the magnetic field and vibration negatively. That's why it is crucial to implement a Kalman filter. Kalman filter is a quite applicable method for the noisy sensors. This digital filter can be applied to control systems because it also minimizes square error [16]. Noisy signals make difficult to have a balance control. Balance control is more sensitive than other types of controls because when it failed, the body falls down and camera misses the target. The mathematical equations are based on the mathematical model of the system. In conventional Kalman filtering, it is necessary to know the dynamic model of the current system. $\hat{x}_{k}$ and $Z_{k}$ represent state vector and measurement vector respectively and [2]. Kalman filter consist of two phases, including predict and update phases. Equation 6,7 are belonging to predict steps and equation 8, 9, 10 belonging to update steps. Update steps use predict step parameters as inputs.

$\hat{x}_{k}=A \hat{x}_{k-1}+B u_{k-1}+w_{k-1}$

$Z_{k}=H x_{k}+v_{k}$

$u_{k}$ represents the control signal and random variables $w_{k}$ and $v_{k}$ represent the process and measurements noises.
$\mathrm{Z}$ represents the sensor measurements. A, B and $\mathrm{H}$ state matrix.

Below equation is the project error covariance;

$$
\hat{P}_{k}=A P_{k-1} A^{T}+Q
$$

$\mathrm{K}_{\mathrm{k}}$ is the Kalman gain.

$$
K_{k}=P_{k}^{-} H^{T}\left(H P_{k}^{-} H^{T}+R\right)^{-1}
$$

Below equation updates the estimate with $Z_{k}$ measurement.

$$
\hat{x}_{k}=\hat{x}_{k}^{-}+K_{k}\left(z_{k}-H \hat{x}_{k}^{-}\right)
$$

Noise covariance ' $Q$ ' and the measurement noise covariance ' $R$ ' are assumed as constant values. When these values update themselves, filter turns into an adaptive filter.

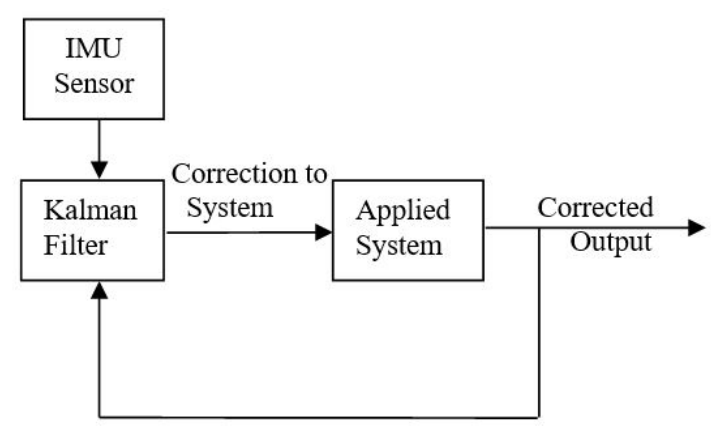

Figure 7. Kalman Filter Block Diagram

Knowledge about systems dynamic model is quite necessary for Kalman filter [3]. As it can be seen clearly from Figure 7. block diagram, Kalman filter uses IMU sensor data as an input. The filter predicts and updates angle. After this stage, Kalman output was sent to the system directly [3]. The filter updates and minimize error by feedbacks. Thanks to this infinite loop, Kalman parameters updates itself. These updates keep the mass in upright position against distortion.

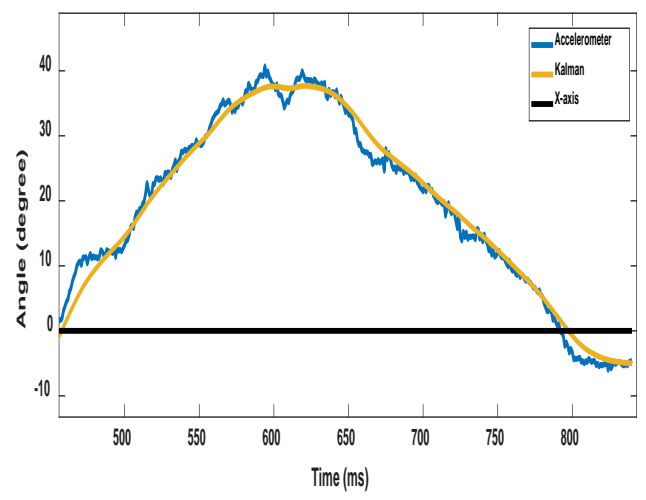

Figure 8. Kalman \& Accelerometer Outputs 


\subsection{PID Controller}

PID controller which is known as the combination of proportional (P), integral (I) and derivative (D) controllers. PID control is one of the most common control methods in the industry because of its easy applicable structure. It doesn't require the mathematical model of the system unlike some modern control system like a Linear Quadratic Regulator (LQR).

PID controllers are first used in mechanical systems. After its high performance in mechanic systems, it was started to use it in electrical systems [5]. As industry improved, the necessity of control was increased. The easy implementation of software made PID controller more interesting for the engineering. One of the important feature of PID controllers is that it manipulates the process inputs according to the change of the signal, in another word it evaluates the error which is the difference between the setpoint and current angle. In the control of balance robot, PID control method was used to maintain the balance $[14,16]$.

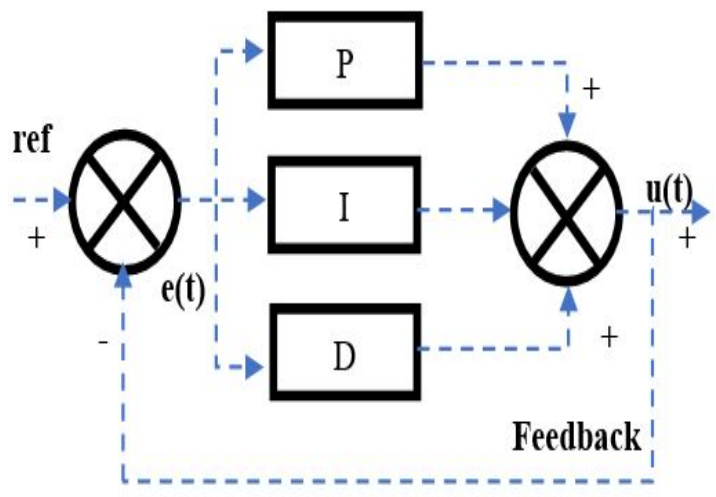

Figure 9. PID Block Diagram

As shown in the above figure, e represents the error of the system which is the difference between reference and output, u represent the output signal. Closed loop control systems have a feedback block. Feedback block helps to update the error and this is quite useful to have a better control. In this figure, error fed into the PID controller. Below Equations show the mathematical representation of PID terms.

$$
\begin{aligned}
& \text { Pterm }=K_{p} e(t) \\
& \text { Iterm }=K_{i} \int_{0}^{t} e(t) d \tau \\
& \text { Dterm }=K_{D} \frac{d e(t)}{d t} \\
& \text { error }=r e f-u(t) \\
& u(t)=K_{p} e(t)+K_{i} \int_{0}^{t} e(t) d \tau+K_{D} \frac{d e(t)}{d t}
\end{aligned}
$$

The mathematical equation of the PID controller can be seen in the equation (15) [29]. Figure 10. helps to understand rise time, settling time and overshoot parameters visually. A represents the rise time. C represents the overshoot and $\mathrm{B}$ represents the settling time.

Having small values of rising time, overshoot and settling time increase the quality of the balance. The control signal must catch the reference signal. Most of the times, it is expected to have a zero steady state error [30-33].

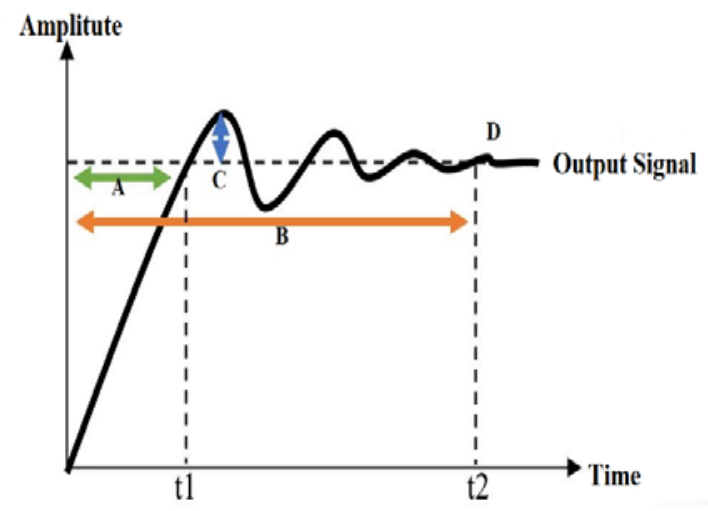

Figure 10. Physical Effects of Control Parameters

The system aims to make the error zero. Table 2. shows the physical effect of $K_{P}, K_{I}$ and $K_{D}$ terms.

Table 2. PID Effects on Control Parameters

\begin{tabular}{|l|l|l|l|}
\hline $\begin{array}{l}\text { Increasing } \\
\text { Parameters }\end{array}$ & \multicolumn{1}{|c|}{$\mathrm{K}_{\mathrm{P}}$} & $\mathrm{K}_{\mathrm{I}}$ & $\mathrm{K}_{\mathrm{D}}$ \\
\hline Rise Time & Decrease & Decrease & $\begin{array}{l}\text { Small } \\
\text { effect }\end{array}$ \\
\hline Over shoot & Increase & Increase & Decrease \\
\hline $\begin{array}{l}\text { Settling } \\
\text { Time }\end{array}$ & $\begin{array}{l}\text { Small } \\
\text { effect }\end{array}$ & Increase & Decrease \\
\hline Steady-State & Decrease & $\begin{array}{l}\text { Big } \\
\text { effect }\end{array}$ & $\begin{array}{l}\text { Small } \\
\text { effect }\end{array}$ \\
\hline
\end{tabular}

Those effects are gained by experimental results of PID controllers. This table is quite useful while tuning the PID manually. Increasing $K_{P}$ and $K_{I}$ terms improves the rise time which is quite important for balance robots. But it has negative effects on overshoot and settling time. Increased overshoot prevents the robot to stay stable and increased settling time means robot can reach the balance position after a while. Increasing $K_{I}$ will have a positive effect on steady-state error. This is also an important parameter to fix. Increasing $\mathrm{K}_{\mathrm{D}}$ decreases the overshoot and settling time and has small effects on rising time and steady state errors. When all these parameter and conditions considered and PID parameter were chosen [33-36]. 


\subsection{Color Detection}

Color detection is one of the ways of object recognition. Objects can be recognized because of their color [30]. Color detection is the visual part of our system. The importance of this part is quite high. In this stage, direction control of the robot was determined and processed. Thanks to the MATLAB \& Arduino interface, it is possible to process an image and control motors. RGB color detection is a quite easy task to process via image acquisition and image processing toolbox[17-19].

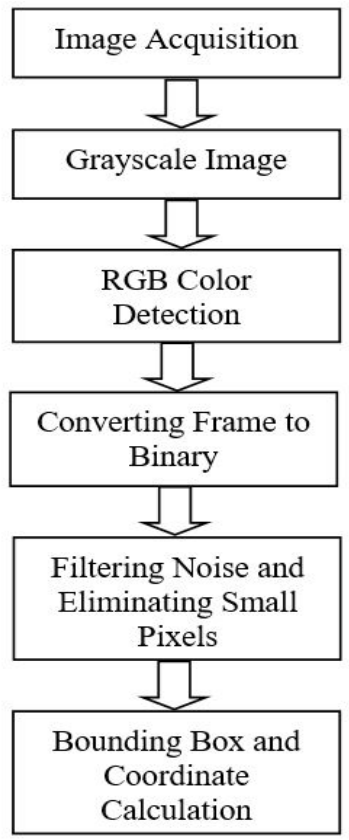

Figure 11. Block Diagram of Object Detection Algorithm

Figure 11 shows the steps for color detection algorithm. It starts with the introducing the camera to the computer as a video object. The camera takes snap-shot of real-time video. The algorithm converts the snapshot image to grayscale image to start the process. The software determined the RGB object and eliminate the noise by using a median filter. After all, it computes the place of the object on the screen in $\mathrm{x}$ and $\mathrm{y}$ axis. This is where we read the motion information of the object [19-21]. If the object moves to the left on the screen, it means object moves to the right in real time. This situation can be explained by the mirror effect [21-24]. Below equations belonging to color detection in an image.

$$
\begin{aligned}
& I(R, G, B)=R+G+B \\
& r(R, G, B)=\frac{R}{R+G+B} \\
& g(R, G, B)=\frac{G}{R+G+B}
\end{aligned}
$$

$b(R, G, B)=\frac{B}{R+G+B}$

' I' represents the intensity, r, g, and b represent the color amount over 255. H (hue) can be calculated with following equation;

$$
H(R, G, B)=\arctan \left(\frac{\sqrt{3}(G-B)}{(R-G)+(R-B)}\right.
$$

Saturation (S) measures the relative white content of a color and it can be calculated in the following equations [25-27];

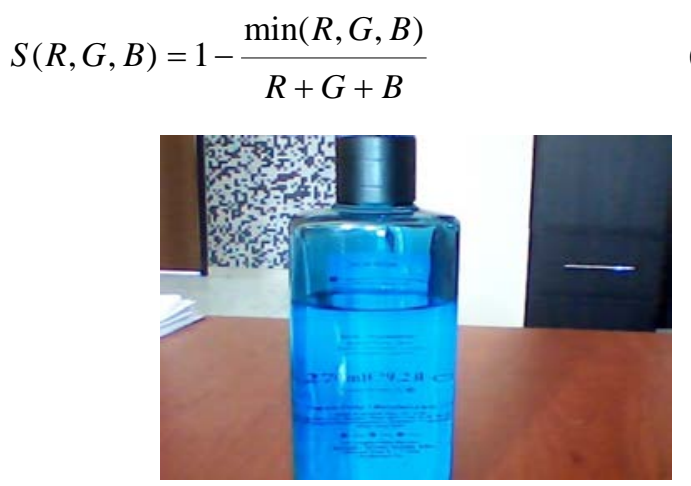

Figure 12. A Blue Object in Visual Field of Camera

Figure 12 shows a blue object in the visual field of the camera. In this stage, it is aimed to point out blue objects in the image. By using above algorithm in Figure 11, the system eliminates the non-blue parts of the image. This can be seen in Figure 13.

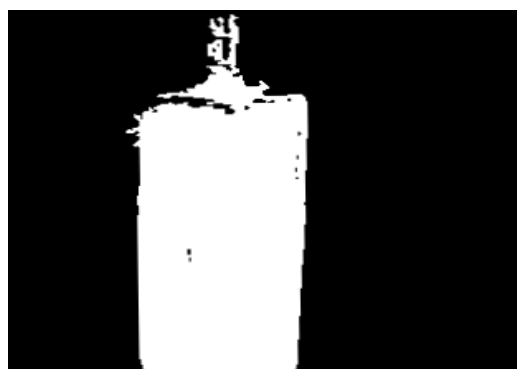

Figure 13. Detected Color in the Image

After the elimination of non-blue parts, we compute the coordinates of blue objects in the image. This can be seen in Figure 14.

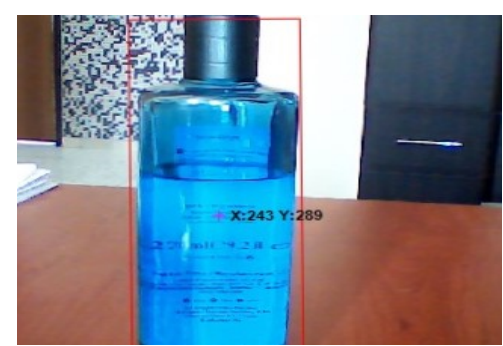

Figure 14. Coordinates of the Object 
Now we have the central coordinates of a bounding box of blue objects in the image. This information update itself when the object moves and this inform us about the motion information of objects. This information was used in the direction control of robot mass[27, 28].

\section{RESULTS}

This paper presents the design of an autonomous robot which can track colorful objects while balancing itself on two wheels at the same time. Figure 15 shows the top and side view of the robot. Table 3 shows the commands and object positions together. Computer perceives the object position from its central coordinates.

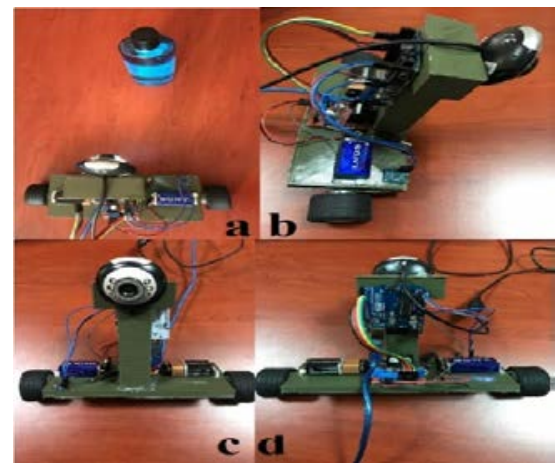

Figure 15. Robot Body

Number 1 situation illustrates where the robot goes back. In this illustration, 2 motors turn to go back without losing balance control. Similarly, in the second illustration, the right motor goes forward while the left motor is stable. This action turns the mass to the left. In the third illustration, the left motor goes forward while the right motor is stable and this action turns the mass to the right. In the last illustration, motors go forward without losing balance control.

Table 3. Direction Control According to Motion Information

\begin{tabular}{|c|c|c|}
\hline & Object Positions & $\begin{array}{c}\text { Track } \\
\text { Command }\end{array}$ \\
\hline 1 & & Go \\
\hline 2 & & back \\
\hline 3 & & Turn \\
& & Teft \\
& &
\end{tabular}

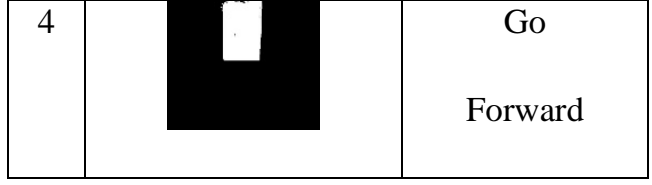

Figure 16 shows a graph belonging to the robot's IMU sensor for experiment 1.a. Accelerometer signal which is represented with the blue signal has plenty of noise and vibration. After implementation of Kalman filtering, it is more stable and less noisy and represented with the orange signal. 1.5 second after the robot starts to stay in balance, the body was disturbed.

Between 1.5-2 seconds robot achieved to get back balance position. After 2 seconds, the body is in balance position again. In this experiment, PID parameter was adjusted like following $K_{P}=300, K_{I}=1, K_{D}=70$. It is quite clear that the degree signal of the robot has lots of overshoot because of the high value of $K_{p}$. As it was stated above, having a high value of $K_{P}$ and $K_{I}$ increase the overshoot.

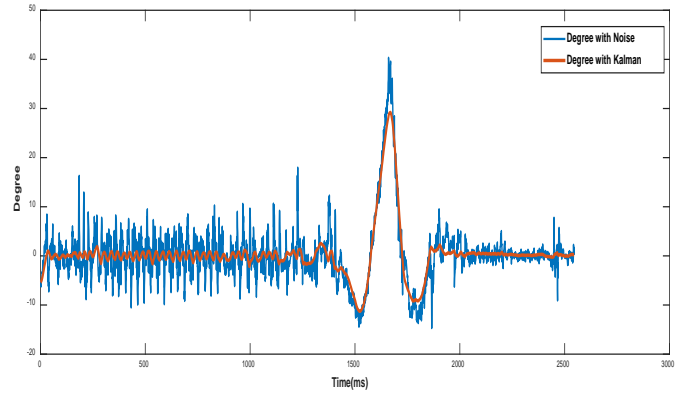

Figure 16. Experiment 1.a IMU Signal

The control signal of experiment 1.a can be seen in Figure 17. Between 1.5 and 2 seconds, motors were driven with max PWM value (255) to get back balance position as it should be.

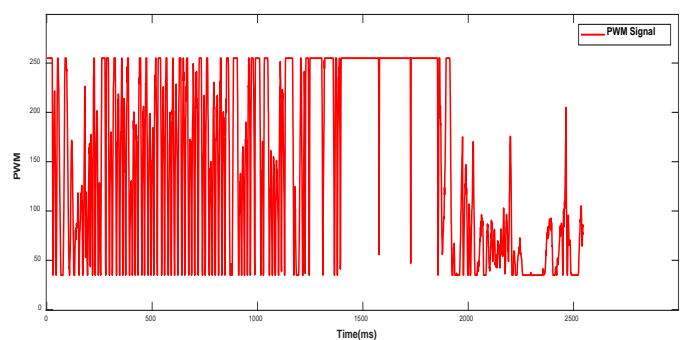

Figure 17. Experiment 1.a Control Signal

In the experiment 2.a PID parameters were adjusted with following values $K_{P}=30, K_{I}=10, K_{D}=70$. IMU signal of the robot has lots of noise and vibration like the previous experiment.

$K_{P}$ was decreased but $K_{I}$ was increased so increasing $K_{I}$ also effects overshoot. When it compared with the previous experiment, total overshoot is less. This situation also affects the control signal in Figure 19. 


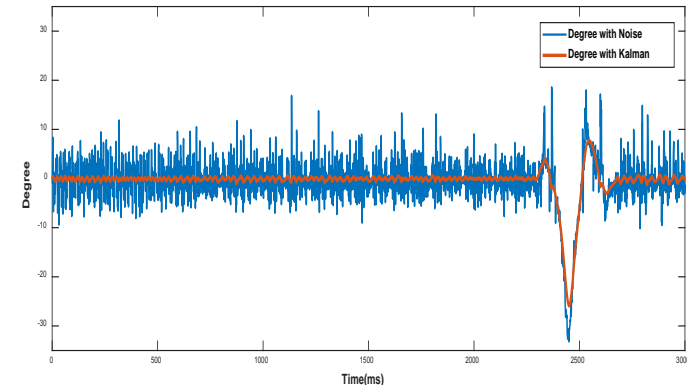

Figure 18. Experiment 2.a IMU Signal

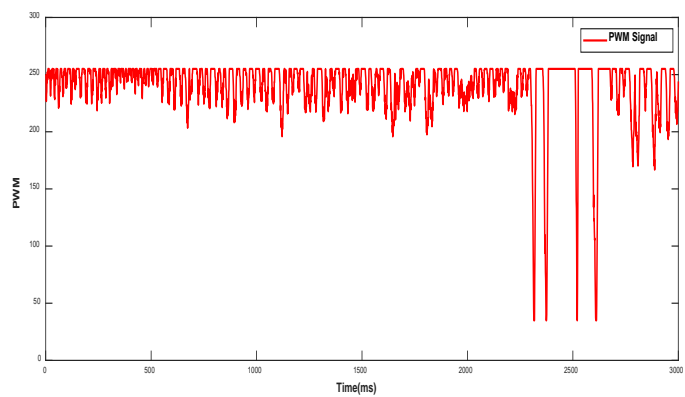

Figure 19. Experiment 2.a Control Signal

In the last experiment, PID parameters were adjusted with following values $K_{P}=90, K_{I}=0.5, K_{D}=0$. Increasing $K_{I}$ affects overshoot parameters positively. In balance position, control signal value is less than 255. The control signal is more stable for these parameters.

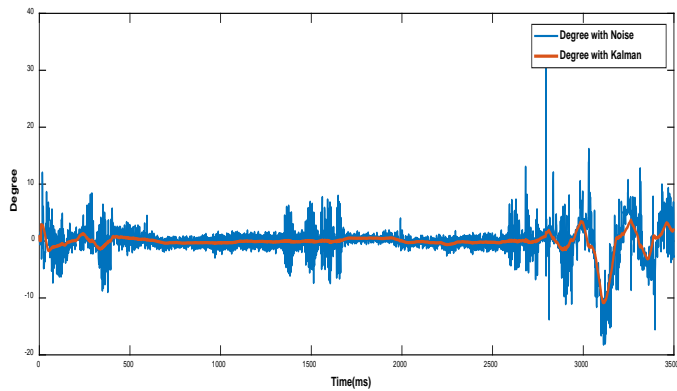

Figure 20. Experiment 3.a IMU Signal

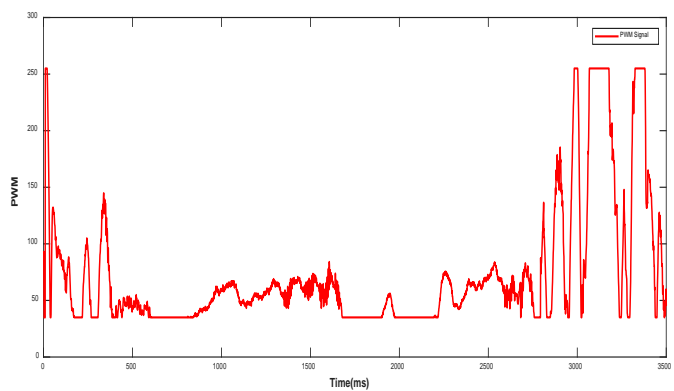

Figure 21. Experiment 3.a Control Signal

\section{CONCLUSIONS}

This paper presents a real-time robot which can track a colourful object and stand in upright position simultaneously. A physical model of the robot and necessary hardware were presented. Balance control was managed by using PID control. The effect of PID values over PID parameters were discussed. Optimal PID values were determined by trial and error method. The output of IMU sensor was also presented. The problems of IMU sensors were solved by using a Kalman Filter. Moreover, a color detection algorithm was presented and explained with block diagrams and process steps. A blue object was chosen as an object to track. According to motion information of the object, motors were driven to keep the object in the visual field of the camera. During the construction of this autonomous robot, many methods were learned and practiced.

\section{ACKNOWLEDGEMENT}

The author wishes to thank The Dean of Architecture and Engineering Faculty of Kahramanmaras Sutcu Imam University "Mahit Gunes" because of his technical supports.

\section{REFERENCES}

[1] Y. Ding, J. Gafford, and M. Kunio, "Modeling, Simulation and Fabrication of a Balancing Robot", Advanced Dynamics and Control, vol. 151, p. 22, 2012.

[2] A. Fakharian, T. Gustafsson, and M. Mehrfam, "Adaptive Kalman filtering based navigation: An IMU/GPS integration approach", IEEE International Conference on Networking, Sensing and Control, 11-13 April, Delft, Netherlands, pp. 181-185, (2011).

[3] R. C. Ooi, "Balancing a two-wheeled autonomous robot", Final Year Thesis, School of Mechanical Engineering, University of Western Australia, vol. 3, 2003. [4] A. Castro, "Modeling and dynamic analysis of a twowheeled inverted-pendulum", Master Thesis, Mechanical Engineering, Georgia Institute of Technology, Georgia, 2012.

[5] A. Gani, E. Kılıç, Ö. F. Keçecioğlu, H. Açıkgöz, And M. Şekkeli, "PID And Fuzzy Logic Controller Design For The Level And Temperature Control Of Mixing Tank Used In Industrial Applications", Engineer \& the Machinery Magazine, vol.57, no. 675, pp. 41-48, 2016.

[6] H. Açıkgöz, Ö. F. Keçecioğlu, M. Güneş, and M. Şekkeli," Simulation Study of Hydraulic Turbine by Using Self-Tuning Fuzzy PID Controller", Academic PlatformJournal of Engineering and Science, vol. 3, no. 1, pp. 7-15, 2015.

[7] A. Gani, O. F. Kececioglu, H. Acikgoz, and M. Sekkeli,"Fuzzy Logic Controller Design Based On Sugeno Inference Method For Nonlinear Inverted Pendulum Dynamical System", Sigma Journal Of Engineering And Natural Sciences-Sigma, Vol. 8, No. 1, Pp. 19-30, 2017.

[8] C. Sundin and F. Thorstenson, "Autonomous balancing robot: Design and construction of a balancing robot", Master Thesis, Mechanical Engineering Chalmers University Of Technology, Goteborg, Sweden, 2012.

[9] S. A. Junoh, "Two-wheeled balancing robot controller designed using PID", Master Thesis, Faculty of Electrical 
and Electronic Engineering, Universiti Tun Hussein Onn Malaysia, 2015.

[10] M. A. Şen, "Design And Optimisation Of A Fuzzy Logic Based Controller For A Two-Wheeled Robot By Using The Bees Algorithm", Master Thesis, Institution of Science and Technology, Selcuk University, Konya, 2014. [11] A. Ünlütürk, U. Güner, And Ö. Aydoğdu," A New PIV Type Controller Design and Its Application on Balance Robot",TOK Conference, (September 11-13, Kocaeli, Turkey) pp. 578-582, (2014).

[12] H.-C. Sung, "Balancing Robot Control and Implementation", Master Thesis, Department of Mechanical Engineering Texas A\&M University, 2015.

[13] H. Guducu, "Building Detection from Satellite Images Using Shadow and Color Information", Master Thesis, Electrical And Electronics Engineering Natural And Applied Sciences Of Middle East Technical University, Texas, 2008.

[14] S. Balasubramanian and M. N. Lathiff, "Self balancing robot", Undergraduate Thesis, Engineering Physics Project Laboratory, The University Of British Columbia, Vancouver, 2011.

[15] O. Enginoğlu, "Design and control of balancing robot", Master Thesis, DEÜ Institue of Scince and Engineering, 2012.

[16] J. L. C. Miranda, "Application of Kalman Filtering and PID Control for Direct Inverted Pendelum Control", Master Thesis, Electrical and Computer Engineering, California State University, California, 2010.

[17] M. S. Uzuner, N. Yilmaz, And M. Bayrak, "A RealTime Tracking Application Of Different Coloured Objects with A Vision Based Mobile Robot", Journal of Engineering and Architecture of Gazi University, Vol. 25, No. 4, 2010.

[18] S. Dutta and B. B. Chaudhuri, "A color edge detection algorithm in RGB color space", International Conference on, IEEE Advances in Recent Technologies in Communication and Computing, 27-28 October, Kottayam-India, pp. 337-340, (2009).

[19] V. Kravtchenko, "Tracking color objects in real time", Master of Science, Computer Science, University of British Columbia, Vancouver, 1999.

[20] T. Şentürk, "Real Time Object Tracking with Moving Camera", Master of Science, Computer Science, Institue of Scince and Engineering YTU, Istanbul, 2008.

[21] H. Takemura, K. Ito, and H. Mizoguchi, "Person following mobile robot under varying illumination based on distance and color information", IEEE International Conference on Robotics and Biomimetics, 15-18 December, Sanya-China, pp.1500-1505, (2007).

[22] M. S. Uzer, " A Tracking Application of Different Coloured Targets With A Vision Based Mobile Robot", Master Thesis, Institue of Scince and Engineering Selcuk University, Konya, 2008.

[23] B. Karasulu, "Review and evaluation of well-known methods for moving object detection and tracking in videos", Journal of aeronautics and space technologies, vol. 4, no. 4, pp. 11-22, 2010.
[24] T. Gevers and A. W. Smeulders, "Color-based object recognition", Pattern recognition, vol. 32, no. 3, pp. 453464, 1999.

[25] Y. Çelik, M. Altun, and M. Güneş, "Color based moving object tracking with an active camera using motion information", Artificial Intelligence and Data Processing Symposium (IDAP), 16-17 September, Malatya-Turkey, pp. 1-4, (2017).

[26] M. F. Aslan, A. Durdu, and K. Sabanci, "Shopping Robot That Make Real Time Color Tracking Using Image Processing Techniques", International Journal of Applied Mathematics, Electronics and Computers, vol. 5, no. 3, pp. 62-66, 2017.

[27] W. ladys law Skarbek and A. Koschan, "Colour image segmentation a survey", IEEE Transactions on circuits and systems for Video Technology, vol. 14, 1994.

[28] M. M. Kelek, M. F. Aslan, and A. Durdu, "Real-Time Target Tracking Using Fast Object Detection", International Congress on Engineering and Natural Science, 24-28 May, Sarajevo-Bosnia, pp. 1807-1811, (2016).

[29] A. Kayabası, B. Yıldız, K. Sabanc1, E. Yigit, A. Toktas and M. Tekbas," Colour Feature-Based Classification of Wheat Grain Using ANN with Bayesian Regularization Learning Algorithm",3rd International Conference on Science, Ecology and Technology (ICONSETE), 14-16 August, Rome-Italy, pp. 174-186, (2016).

[30] K. Sabanci, A. Kayabasi, and A. Toktas, "Computer vision-based method for classification of wheat grains using artificial neural network", Journal of the Science of Food and Agriculture, vol. 97, no. 8, pp. 2588-2593, 2017. [31] T. Selcuk and A. Alkan," Pv Modul Simulation And $\mathrm{Pv}$ System Toolbox Implementation In Simulink", Academic Knowledge Conference, 5-7 February, MersinTurkey, pp. 383-386, (2014).

[32] M. Altun, Y. Celik, M. Gunes, "Investigation of The Success of Particle Swarm Optimization Based PID Classic PID and Fuzzy Type Inspection Methods in Speed Control of DC Motor”, Journal of Engineering Science Kahramanmaras Sutcu Imam University, vol. 20, no. 4, pp. 158-167, Dec. 2017.

[33] M. Altun, Y. Celik, M. Gunes," Investigation of The Success Of Pid And Fuzzy Type Inspection Methods In Speed Control Of Shunt Excited Dc Motor", 2nd International Energy and Engineering Conference 12-13 October Gaziantep- Turkey, pp. 451-461, (2017).

[34] O. Doğmuş, E. Kiliç, S. Şit, And M. Güneş,"Adaptation of Optimized PID Controller with PSO Algorithm to Photovoltaic MPPT System", Journal of Engineering Science Kahramanmaraş Sutcu Imam University, vol. 20, no. 4, pp. 1-8, Dec. 2017.

[35] S. Şit, H. R. Özçalik, E. Kiliç, And M. Altun,“ Comparative Study of the Success of PI and PI Fuzzy Controller for Induction Motor Drive using SVPWM Method”, International Journal of Engineering Science Invention (IJESI), vol. 5, no. 11, pp. 105-114, Nov. 2016. [36] A. Gani, E. Kiliç, Ö. F. Keçecioğlu, H. Açikgöz, M. Tekin, And M. Şekkeli”, A Simulation Study on 
Controlling Excitation Current of Synchronous Motor and Reactive Power Compensation via PSO Based PID and
PID Controllers", Conference on Innovations and Applications in Intelligent Systems, 5-7 October, AlanyaTurkey, (2017). 